\title{
Globe
}

Revue internationale d'études québécoises

\section{Jean-Denis Gendron, La modernisation de l'accent québécois. De l'accent traditionnel au nouvel accent, 1841-1960. Esquisse historique, Québec, Presses de l’Université Laval, 2014}

\section{Luc Ostiguy}

Volume 17, numéro 2, 2014

URI : https://id.erudit.org/iderudit/1036246ar

DOI : https://doi.org/10.7202/1036246ar

Aller au sommaire du numéro

Éditeur(s)

Globe, Revue internationale d'études québécoises

ISSN

1481-5869 (imprimé)

1923-8231 (numérique)

Découvrir la revue

Citer ce compte rendu

Ostiguy, L. (2014). Compte rendu de [Jean-Denis Gendron, La modernisation de l'accent québécois. De l'accent traditionnel au nouvel accent, 1841-1960. Esquisse historique, Québec, Presses de l'Université Laval, 2014]. Globe, 17(2), 216-219. https://doi.org/10.7202/1036246ar d'utilisation que vous pouvez consulter en ligne.

https://apropos.erudit.org/fr/usagers/politique-dutilisation/ 
boudé par les historiennes féministes. Baillargeon remédie à cette tendance en abordant celui des femmes œuvrant dans les services de santé, un sujet au cœur de ses recherches depuis plusieurs années. Le rôle des infirmières au sein de la société québécoise, par exemple, trouve sa pertinence dans cette synthèse sur l'histoire des femmes. Habilement, l'historienne participe à la déconstruction du mythe de leur servitude et fait la preuve de leur nouveau militantisme dans les des années 1960 et 1970 : un sujet clairement négligé par l'historiographie jusqu'à la fin des années 1990. Finalement, cette brillante synthèse «montre que le travail, les conditions d'existence, le statut juridique et l'engagement social et politique des femmes ne peuvent se comprendre qu'en référence à la définition sociale de la féminité, elle-même construite de manière interdépendante avec la masculinité, mais aussi à l'appartenance de classe, à l'ethnicité ou à la race qui expliquent la diversité de l'expérience historique féminine» (p. 245)... Une diversité qui retient maintenant l'attention des jeunes chercheuses et chercheurs, particulièrement en ce qui a trait à la diversité sexuelle et à la construction de genre.

Marie-Claude Thifault Chaire de recherche sur la francophonie canadienne en santé Université d'Ottawa

\section{Jean-Denis Gendron}

La modernisation de l'accent québécois. De l'accent traditionnel au nouvel accent, 1841-1960. Esquisse historique, Québec, Presses de l'Université Laval, 2014.

L'essai de Jean-Denis Gendron, La modernisation de l'accent québécois. De l'accent traditionnel au nouvel accent: 1841-1960. Esquisse historique, se présente comme un retour sur les efforts qui, à partir de 1841, ont été consentis par des éducateurs pour corriger la prononciation des Québécois. La période considérée par l'auteur commence en 1841 avec l'ouvrage correctif de Thomas Maguire, Manuel des difficultés plus communes de la langue française, adapté au jeune âge, et suivi d'un recueil de locutions vicieuses, et se termine au début des années 1960 avec la parution de son propre ouvrage correctif, Phonétique orthophonique à l'usage des Canadiens français: orthophonie, exercices de correction, textes de lecture (PUL, 1965). Sur ce plan, l'ouvrage a le mérite de rappeler à la mémoire du lecteur les personnages qui, 
chacun en leur temps, ont cherché à moderniser la prononciation traditionnelle des Québécois en faisant la promotion d'une prononciation "soignée " dans le but avoué de la rapprocher de celle de l'élite française de la même époque, ainsi que leurs ouvrages correctifs, qui ont servi d'outils aux enseignants pour perfectionner la diction de ceux qui constituaient ou allaient constituer l'élite québécoise. Outre Maguire et Gendron déjà évoqués, il y a eu Jérôme Demers, Thomas-Étienne Hamel, Pierre-Minier Lagacé, Adjutor Rivard, Joseph Dumais, Eugène Lassalle, Georges Landreau, Théophile Hudon, Yvonne Duckett-Audet et Robert Dubuc.

L'essai se présente aussi comme une «image» des voyelles et des consonnes et de leur diction qui caractérisaient la prononciation traditionnelle des Québécois, ainsi que celle de son mode d'élocution dans son ensemble (rythme syllabique et intonation) : une image que l'auteur construit à partir de ses connaissances et des remarques des pédagogues et de leurs contenus d'enseignement (chapitres 5 et 6 ).

Pourquoi la prononciation des Québécois devait-elle être «modernisée »? En quoi était-elle si différente de celle des Parisiens, si on se rappelle l'origine toute parisienne de la prononciation du français québécois, dont la plupart des historiens de la langue conviennent? Dans son essai, Gendron reprend l'idée qu'il a exposée dans son précédent ouvrage, D’où vient l'accent des Québécois? Et celui des Parisiens?, paru en 2007 chez le même éditeur, dans lequel il fait valoir que la prononciation de la société parisienne avait diamétralement changé au moment de la Révolution française, début d'une période marquée par une rupture des relations entre le Canada et la France qui a duré jusqu'à la fin des guerres napoléoniennes.

Gendron, s'inspirant des thèses de l'historien de la langue Alexis François, fait valoir que les Parisiens connaissaient, au XVII et au XVIII ${ }^{\mathrm{e}}$ siècle, deux types de prononciation. Il y avait, d'une part, celle de la conversation mondaine chez les courtisans, du discours privé si on peut dire, qui se voulait naturelle et qui était, apparemment, voisine de celle du peuple de Paris. Il y avait, d'autre part, la prononciation cultivée pour le discours public (exposé, plaidoirie, théâtre), calquée en partie sur l'écrit en ce qu'elle faisait entendre bien des lettres qui demeuraient muettes dans la conversation privée et caractérisée par une articulation plus énergique, précise : c'était cette seconde façon de prononcer les sons et les syllabes qui était enseignée dans les collèges, qui est devenue, au cours du XVIII ${ }^{\mathrm{e}}$ siècle, celle de la grande bourgeoisie française qui fréquentait les écoles et qui s'est imposée comme norme à Paris à partir de 1789 avec l'accession au pouvoir de cette bourgeoisie. 
Selon Jean-Denis Gendron, la prononciation entendue en Nouvelle-France aurait été essentiellement celle de la conversation mondaine des courtisans, voulue naturelle et spontanée. La distinction de style entre discours privé et discours public n'aurait pas traversé l'Atlantique, seule la prononciation familière aurait été pratiquée, affirme Gendron sans expliquer (p. 26). Lorsque les Canadiens et les Français ont recommencé à se visiter à partir de 1830, ils ont pris conscience de l'écart qui existait entre leurs modèles de prononciation respectifs (chapitre 1).

C'est à partir de ce moment que des pédagogues québécois se sont donné comme objectif de faire la promotion auprès de l'élite québécoise d'une prononciation "soignée ", inspirée de la prononciation parisienne. Au début, seul le discours public de cette élite était visé ; plus tard, c'était le discours privé de toute la société québécoise, invitée à participer à cette modernisation (chapitre 2). Dans les termes de l'auteur, l'entreprise a réussi en partie « en modifiant considérablement la prononciation des Québécois en la faisant glisser lentement de l'accent originel et de la forme traditionnelle des mots vers des formes et une prononciation modernisées ". C'est cette histoire qui est racontée, que Gendron fait cesser en 1960 avec ses propres travaux de description phonétique des prononciations du français québécois. Comme il le mentionne, l'étude de la période qui suit, de la Révolution tranquille à aujourd'hui, reste à faire.

L'essai apporte donc un éclairage sur une partie de l'histoire du français au Québec en rappelant à notre mémoire ceux qui ont œuvré à l'alignement de la prononciation traditionnelle sur la prononciation parisienne; ceux qui, en dépit des préjugés linguistiques des Québécois à l'égard de l'accent des Parisiens, ont voulu que nos élites prononcent un peu comme les autres élites francophones. L'essai de Gendron s'inscrit ainsi dans les efforts de plusieurs linguistes de construire, selon leurs divers points de vue, une histoire sociolinguistique du français québécois.

Le lecteur non spécialiste de la question sera enchanté par le souci de l'auteur de bien lier les parties de son essai par de constants rappels : l'exposé en est clair et le fil conducteur est constamment ravivé. Il sera aussi ravi d'avoir accès à un même endroit à des extraits des manuels correctifs, dont les contenus constituent des témoignages de prononciations en voie de disparaitre ou déjà disparues, ainsi qu'à des planches anatomiques représentant les sons tels qu'ils sont articulés en français québécois et en français parisien.

Le lecteur spécialiste, quant à lui, trouvera aussi son compte en ce qui concerne l'histoire de ceux qui ont participé à la modernisation de l'accent québécois, mais ne trouvera rien de vraiment nouveau au sujet des 
prononciations elles-mêmes, la plupart ayant fait l'objet d'études ou de mentions dans des publications spécialisées. Il pourrait même être étonné de ne voir aucune référence à celles-ci.

Gendron suggère que, au début du XIX siècle, l'accent traditionnel était largement partagé par tous les Québécois de statuts sociaux différents. Pourtant, on peut présumer qu'il y avait tout de même des prononciations en concurrence. Par exemple, la concurrence entre les prononciations [wa] et [we] - [we] du graphème $o i$, emblématique de la France du XVIII ${ }^{e}$, qui a créé une polémique entre Maguire et Demers (p. 15), a-t-elle vécu dans la vallée laurentienne? L'auteur n'analyse pas les données linguistiques dans ce cadre. Son choix s'explique sans doute par la difficulté du projet et par la nature même de son corpus, à savoir les remarques des pédagogues qui, de par leurs visées, ne signalaient que les prononciations qui étaient différentes de celles du français parisien moderne.

Luc Ostiguy

Université du Québec à Trois-Rivières

\section{Andrée Rivard \\ Histoire de l'accouchement dans un Québec moderne, Montréal, Les Éditions du remue-ménage, 2014.}

Réfléchir à la médicalisation de la naissance, c'est chercher à comprendre comment la science s'est progressivement emparée de l'une des expériences humaines les plus fondamentales, sans égard au fait que la grande majorité des femmes enceintes soient en santé ou qu'elles aient une grossesse «normale». Ce livre, issu des recherches doctorales de l'auteure, s'inscrit dans le champ des recherches critiques et des réflexions féministes, d'où son postulat de départ voulant que les femmes, actrices de premier plan, soient "porteuses du changement social» (p. 25). Pierre de touche de la thèse mise de l'avant, ce changement concerne l'émergence de nouvelles attentes individuelles quant aux conditions de mise au monde qui se manifestent, à partir des années 1960, "par une affirmation sans précédent des droits des femmes" (p. 129). Sur le plan théorique, l'auteure mobilise les travaux du sociologue Alain Touraine sur la modernité ainsi que la perspective foucaldienne sur le biopolitique pour analyser les rapports que les femmes entretiennent avec les appareils médical et étatique sur l'accouchement, et ce, 